Ann. Sci. forest., 1977, 34 (1), 47-57.

\title{
Effets des poussières sur la photosynthèse
}

\section{Influence des polluants particulaires sur la photosynthèse du Pin sylvestre et du Peuplier}

\author{
D. AUCLAIR \\ Station de Recherches sur la forêt et l'environnement, Centre de Recherches forestières d'Orléans, I.N.R.A., \\ Ardon, 45160 Olivet
}

\begin{abstract}
Résumé
Dans le but d'étudier les effets physiques des particules solides sur la végétation, des mesures de photosynthèse en enceinte contrôlée ont été effectuées sur des plants de Pin sylvestre et de Peuplier, avant et après empoussièrement.

Les résultats ont pu être illustrés en ajustant la courbe de réponse en fonction de l'éclairement au modèle schématique $m \mathrm{P}^{2}-\left(\alpha E+\mathrm{P}_{\infty}\right) \mathrm{P}+\alpha E \mathrm{P}_{\infty}=0$.

Aux éclairements élevés n'apparaît pas d'inhibition, le Peuplier semble au contraire présenter une assimilation plus élevée lorsqu'il est empoussiéré. Par contre, aux éclairements faibles, les poussières entraînent une nette diminution de l'assimilation de $\mathrm{CO}_{2}$. Ce résultat est confirmé par l'étude des propriétés spectrales des poussières : celles-ci interceptent une partie de l'énergie lumineuse, n'en transmettant qu'une fraction, le rendement lumineux de la photosynthèse aux faibles éclairements incidents s'en trouve donc réduit.
\end{abstract}

\section{Introduction}

Les effets des polluants particulaires sur la végétation, et en particulier sur la photosynthèse, ont été étudiés depuis quelques années par plusieurs chercheurs aux Etats-Unis et en Allemagne surtout, dans le but de procurer des normes de qualité de l'air. Ces études ont toujours porté sur l'effet à long terme d'une poussière de provenance, et donc de composition chimique, déterminée. Elles ont été rassemblées par Darley (1966) et par Auclair (1976a) dans des revues bibliographiques.

Dans une étude précédente (Auclair, 1976b), nous avons étudié l'effet à long terme d'un empoussièrement contrôlé de poussière de charbon et de ciment sur la photosynthèse d'Epicéas. Nous cherchons ici à approfondir cette étude, ne tenant compte que de l'effet physique des poussières sur la photosynthèse, qui sera donc généralisable à des poussières de composition chimique différente. Dans cet esprit, nous avons cherché à mettre en évidence, d'une part un éventuel effet " mécanique» sur le mécanisme stomatique par exemple, d'autre part une éventuelle modification de l'énergie lumineuse ou des propriétés optiques des feuilles par la couche de poussières se trouvant à la surface des feuilles. 


\section{Matériel et méthodes}

Des expériences identiques ont été effectuées en 2 temps, d'une part sur du Pin sylvestre et d'autre part sur du Peuplier :

- en avril les mesures ont été faites sur Pinus sylvestris (L.) de provenance Alsace (65 600) (Station d'Amélioration des Arbres forestiers, I.N.R.A.). Des plants de 2 ans ont été cultivés en serre à partir de janvier afin de les obtenir dans des conditions de développement suffisamment avancées au moment de l'essai ;

- en juin, les mesures ont été effectuées sur Populus euramericana (clone I 214) cultivés 5 à 6 semaines sous conditions contrôlées : photopériode de 15 heures à 6000 lux, et thermopériode $27^{\circ} \mathrm{C} / 24^{\circ} \mathrm{C}$ (Duval, 1975).

Les mesures suivantes sont effectuées sur chaque plant :

$1^{\circ}$ a) Mesures de la photosynthèse nette en enceinte contrôlée (Siemens) par analyse différentielle infrarouge à 5 éclairements (éclairage artificiel par lampe au mercure Mazda), à l'aide de l'appareillage décrit par Duval (1975). Les conditions expérimentales à l'intérieur de l'enceinte sont :

- température constante $20^{\circ} \mathrm{C} \pm 1^{\circ} \mathrm{C}$,

- humidité constante 71 p. $100 \pm 3$ p. 100 ,

- vitesse de turbulence de l'air 1 mètre/seconde environ,

- renouvellement d'air dans l'enceinte, environ 10 minutes.

Un rameau de Pin sylvestre ou une tige de Peuplier (environ 4 feuilles), est placé(e) à l'intérieur de la chambre, les racines étant bien alimentées en eau (à la capacité au champ).

b) La durée des mesures de la photosynthèse à 5 éclairements étant d'une demi-journée, un plant mesuré le matin (resp. l'après-midi) est remis en serre, puis le lendemain est empoussiéré en début de matinée (resp. d'après-midi), à l'aide d'une trompe supersonique Bertin (Plaisir, France). Une poussière de charbon relativement uniforme est ainsi obtenue. L'examen au microscope optique de cette poussière a montré que le diamètre apparent des particules était compris entre 1 et 20 microns. Ce dispositif permet d'obtenir des empoussièrements voisins des empoussièrements naturels.

c) Aussitôt après empoussièrement, on effectue de nouvelles mesures de photosynthèse, identiques aux précédentes, sur le même rameau.

$2^{\circ}$ Des mesures de transpiration et de température foliaire ont été effectuées pour mémoire, mais n'ont donné aucun résultat significatif, du fait de la trop grande dispersion des résultats, due à l'appareillage utilisé.

$3^{\circ}$ Les mesures de photosynthèse sont rapportées à la masse de matière sèche photosynthétisante et, pour le Peuplier, à la surface foliaire. Celle-ci est mesurée à l'aide d'un appareil Milliplan (Metraplan). On ne tient compte que d'une face, la face inférieure n'étant que très peu éclairée par un rayonnement diffus. Compte tenu de la structure des aiguilles de conifères, nous n'avons pas mesuré leur surface.

Le dosage des poussières se fait en lavant les feuilles dans de l'eau, en filtrant cette eau et en pesant les poussières recueillies, après dessiccation à l'étuve. On en déduit ainsi la masse de poussière par unité de surface foliaire (ou de masse d'aiguilles), en tenant compte du taux de récupération (solubilité des poussières). 
$4^{\circ}$ La mesure des propriétés spectrales des feuilles propres ou empoussiérées, ainsi que des poussières seules, a été effectuée à l'aide d'un spectroradiomètre enregistreur ISCO relié par une fibre optique à un microscope ou à une loupe binoculaire. Ce montage a été décrił précédemment par Auclair (1974).

\section{Résultałs}

Les mesures de photosynthèse ont permis d'évaluer, pour un même plant :

- la photosynthèse nette à 5 éclairements, que nous avons calculée en pourcentage par rapport à la photosynthèse maximale, pour le plant propre ;

- la photosynthèse nette du plant empoussiéré, dans des conditions identiques, exprimée en pourcentage par rapport à la photosynthèse maximale du même plant propre.

Les résultats sont portés sur les tableaux 1 et 2 . Toutes les mesures concernant l'énergie lumineuse sont rapportées au spectre total.

\section{TABLEAU 1}

Photosynthèse des Pins sylvestres propres ou empoussiérés, en pourcentage

(poussière de charbon, environ $10 \mathrm{mg} / \mathrm{g}$ de feuilles)

Conditions expérimentales : température, $20^{\circ} \mathrm{C}$; humidité, 71 p. 100

Photosynthesis of clean and dusted Scots Pine

(percentage of maximum photosynthesis of clean plantlet)

Coal dust, ca. $10 \mathrm{mg} / \mathrm{g}$ dry waight

\begin{tabular}{|c|c|c|c|c|c|}
\hline Eclairement $\left(\mathrm{W} . \mathrm{m}^{-2}\right)$. & 30 & 50 & 100 & 180 & 420 \\
\hline Photosynthèse propre (p. 100)... & 14 & 39 & 69 & 90 & $100\left({ }^{1}\right)$ \\
\hline Photosynthèse sale (p. 100). & $\begin{array}{l}* \\
8\end{array}$ & $\begin{array}{l}* \\
32\end{array}$ & $\begin{array}{l}* \\
64\end{array}$ & 88 & 98 \\
\hline
\end{tabular}

* Résultats significativement différents à la probabilité 0,05 .

Statistically significant differences (probability 0.05 ).

(1) $100 \mathrm{p} .100=$ photosynthèse maximale du plant propre. Valeur moyenne $6,7 \mathrm{mg} \cdot \mathrm{g}^{-1} \cdot \mathrm{h}^{-1}$.
(1) $100 \mathrm{p} .100$ maximum photosynthesis of clean plantlet, mean value $6.7 \mathrm{mg} \cdot \mathrm{g}^{-1} \cdot \mathrm{h}^{-1}$.

Les résultałs numériques des mesures de photosynthèse ont fait l'objet d'une étude statistique d'ajustement non linéaire (Roux et Tomassone, 1973-1974). Nous avons cherché à approcher la courbe de réponse à l'aide du modèle de l'hyperbole non équilatère :

$$
m P^{2}-\left(\alpha E+P_{\infty}\right) P+\alpha E P \infty=0 .
$$

Ce modèle est inspiré par Chartier (1966) avec :

- $P=$ photosynthèse brute (nous avons mesuré la photosynthèse nette $P_{N}$, nous avons donc $P=P_{N}-R$, en tenant compte de la respiration que nous avons choisie indépendante de la lumière pour simplifier le modèle) ;

- Les échanges gazeux sont exprimés en pourcentage de la photosynthèse à éclairement maximal du plant avant empoussièrement ; 
- E est l'énergie lumineuse incidente (exprimée en $\mathrm{W} \cdot \mathrm{m}^{-2}$ ) ; mesurée sur le spectre total ;

- $\alpha$ représente le rendement lumineux maximal aux faibles éclairements incidents ;

- $P \infty$ représente la photosynthèse brute à éclairement infini ;

- m est un facteur de correction permettant de tenir compte de la concavité de la courbe (dans le cas de l'hyperbole équilatère, $m=0$ ). Ce facteur dépend en particulier de la résistance à la carboxylation (Chartier, 1966).

\section{TABLEAU 2}

Photosynthèse des Peupliers propres ou empoussiérés, en pourcentage

(poussière de charbon, environ $2 \mathrm{~g} \cdot \mathrm{m}^{-2} \# 80 \mathrm{mg} \cdot \mathrm{g}^{-1}$ )

Conditions expérimentales : température, $20^{\circ} \mathrm{C}$; humidité, 71 p. 100

Photosynthesis of clean and dusted Poplar

(percentage of maximum photosynthesis of clean plantlet)

Coal dust, ca. 2 g. $\mathrm{m}^{-2} \# 80 \mathrm{mg} \cdot \mathrm{g}^{-1}$

\begin{tabular}{|c|c|c|c|c|c|}
\hline Eclairement (W. $\left.\mathrm{m}^{-2}\right) \ldots \ldots \ldots \ldots$ & 25 & 40 & 70 & 130 & 350 \\
\hline Photosynthèse propre (p. 100)..... & $\begin{array}{r}18 \\
*\end{array}$ & $\begin{array}{r}47 \\
*\end{array}$ & 72 & 95 & $\begin{array}{c}100\left({ }^{2}\right) \\
*\end{array}$ \\
\hline Photosynthèse sale (p. 100)........ & 10 & 37 & 64 & 96 & 116 \\
\hline \multicolumn{6}{|c|}{$\begin{array}{l}\text { * Résultats significativement différents à la probabilité } 0,05 . \\
\text { Statistically significant differences (probability } 0.05 \text { ). } \\
\left({ }^{2}\right) 100 \mathrm{p} \cdot 100=\text { photosynthèse maximale du plant propre. Valeur moyenne } 7,3 \mathrm{mg} \cdot \mathrm{dm}^{-2} \cdot \mathrm{h}^{-1} \\
\text { \# } 30 \mathrm{mg} \cdot \mathrm{g}^{-1} \cdot \mathrm{h}^{-1} \text {. } \\
\text { (2) } 100 \mathrm{p} \cdot 100=\text { maximum photosynthesis of clean plantlet ; mean value } 7.3 \mathrm{mg} \cdot \mathrm{dm}^{-2} \cdot \mathrm{h}^{-1} \\
\text { \# } 30 \mathrm{mg} \cdot \mathrm{g}^{-1} \cdot \mathrm{h}^{-1} \text {. }\end{array}$} \\
\hline
\end{tabular}

Nous avons également évalué, à l'aide de ce modèle, la photosynthèse à éclairement maximal $P_{\max }$.

Le faible nombre de points que nous avions pour chaque courbe (5 points par courbe) ne nous a pas permis d'obtenir d'approximations satisfaisantes pour chaque plant considéré séparément: nous n'avons mis en évidence aucune corrélation entre les divers paramètres calculés et la quantité de poussière reçue par chaque plant. $\mathrm{Cecl}$ est dû à la technique utilisée : 5 points pour déterminer 4 paramètres ne peuvent donner que des intervalles de confiance très grands.

Les valeurs moyennes obtenues pour chaque traitement ont été normalisées pour l'étude statistique, elles sont donc données ici en valeur relative :

$$
\begin{array}{rrrl}
\text { Pins sylvestres propres } & \text { Pins sylvestres empoussiérés } \\
\mathrm{R} & =-13,1 \pm 10,7 & \mathrm{R} & =-19,4 \pm 13,0 \\
\alpha & =1,5 \pm 0,9 & \alpha & =1,4 \pm 1,0 \\
\mathrm{P}_{\infty} & =161 \pm 62 & \mathrm{P}_{\infty} & =162 \pm 76 \\
\mathrm{P}_{\max } & =101 \pm 4 & \mathrm{P}_{\max } & =98 \pm 6 \\
\mathrm{~m} & =0,86 \pm 0,12 & \mathrm{~m} & =0,87 \pm 0,14
\end{array}
$$

quantité moyenne de poussière reçue : 10 milligrammes par gramme de feuilles. 


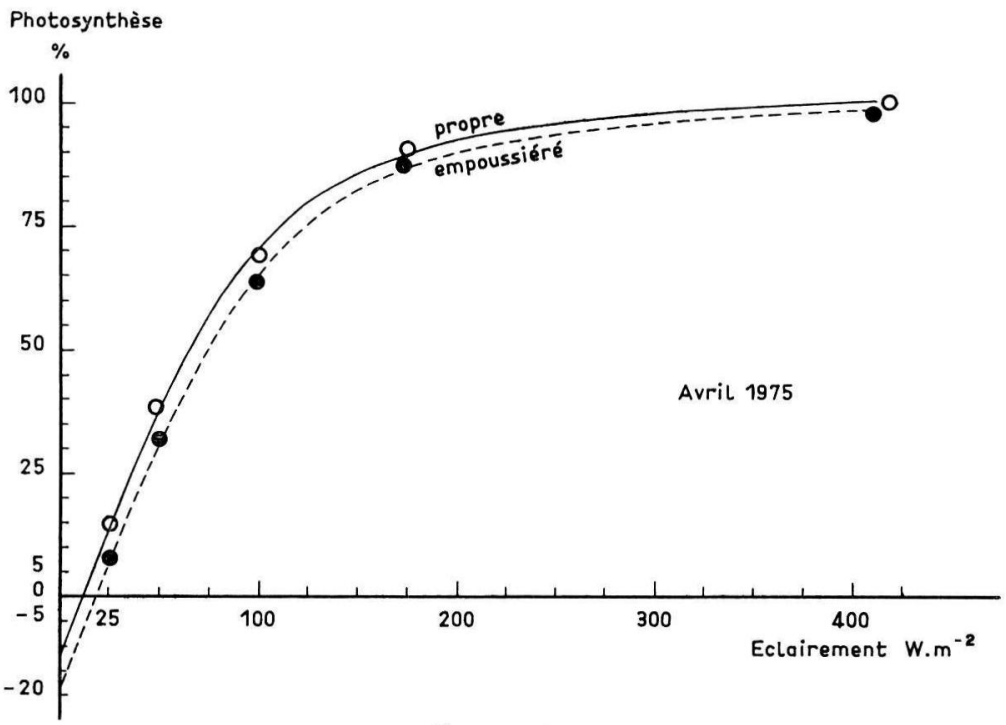

Pin sylvestre

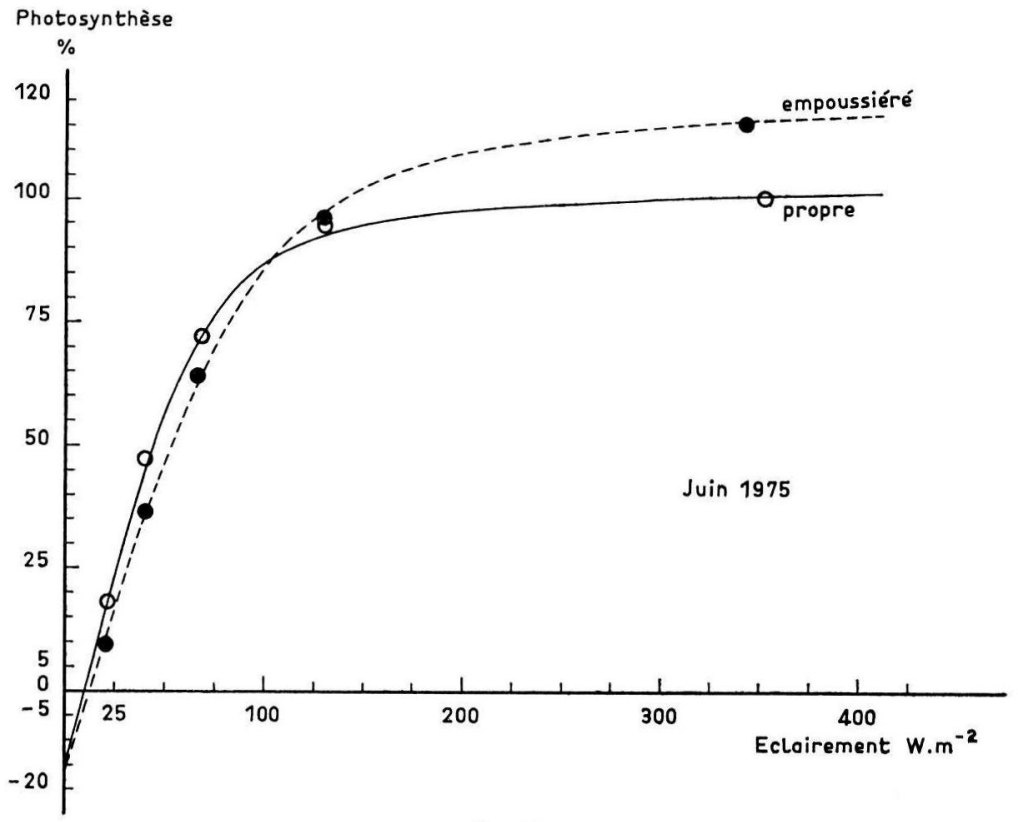

Peuplier

FIG. 1 et 2. - Photosynthèse des Pins sylvestres et des Peupliers.

Photosynthesis of Scots Pine and of Poplar. 


$$
\begin{gathered}
\text { Peupliers propres } \\
\text { R }=-16,5 \pm 16,8 \\
\alpha=2,1 \pm 1,4 \\
\mathrm{P}_{\infty}=149 \pm 61 \\
\mathrm{P}_{\max }=101 \pm 5 \\
\mathrm{~m}=0,90 \pm 0,11
\end{gathered}
$$

Peupliers empoussiérés

$$
\begin{aligned}
\mathrm{R} & =-17,6 \pm 23,0 \\
\alpha & =1,9 \pm 2,1 \\
\mathrm{P}_{\infty} & =186 \pm 143 \\
\mathrm{P}_{\max } & =116 \pm 10 \\
\mathrm{~m} & =0,87 \pm 0,24
\end{aligned}
$$

quantité moyenne de poussière reçue : 2 grammes/mètre carré \#- 80 milligrammes/ gramme de fevilles.

Les courbes correspondantes se trouvent figures 1 et 2 .

On peut remarquer une grande différence entre $P_{\infty}$, l'asymptote horizontale, et $P_{\max }$, la photosynthèse à éclairement maximal calculée par le modèle. Ceci provient de la position des points expérimentaux : il n'y a pas de mesures entre 200 et $400 \mathrm{~W} \cdot \mathrm{m}^{-2}$ pour le Pin ou entre 150 et $350 \mathrm{~W} \cdot \mathrm{m}^{-2}$, pour le Peuplier, ce qui donne une mauvaise approximation de l'asymptote horizontale.
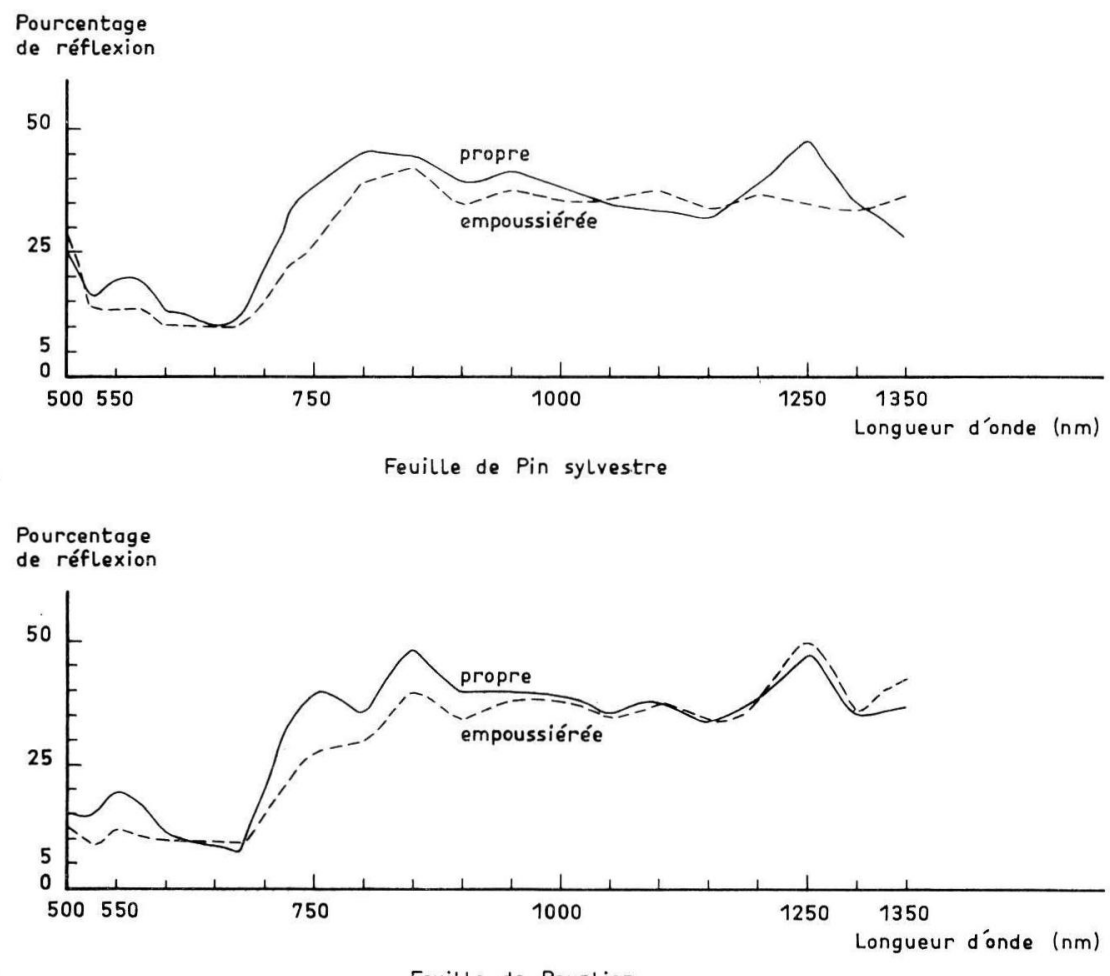

b

Feuille de Peuplier

FIG. 3. - Spectres de réflexion.

a) Feuille de Pin Sylvestre (env. $10 \mathrm{mg} \cdot \mathrm{g}^{-1}$ ).

b) Feville de Peuplier (env. $2 \mathrm{~g} \cdot \mathrm{m}^{-2}$ ).

Reflectance spectra.

a) Leaf of Scots Pine (ca. $\left.10 \mathrm{mg} \cdot \mathrm{g}^{-1}\right)$.

b) Leaf of Poplar (ca. $\left.2 \mathrm{~g} \cdot \mathrm{m}^{-2}\right)$. 
Les spectres de réflexion dans le domaine visible et infrarouge proche, des feuilles de Pin et de Peuplier sont reportés figure 3. Les spectres d'absorption ef de transmission des poussières se trouvent figure 4.

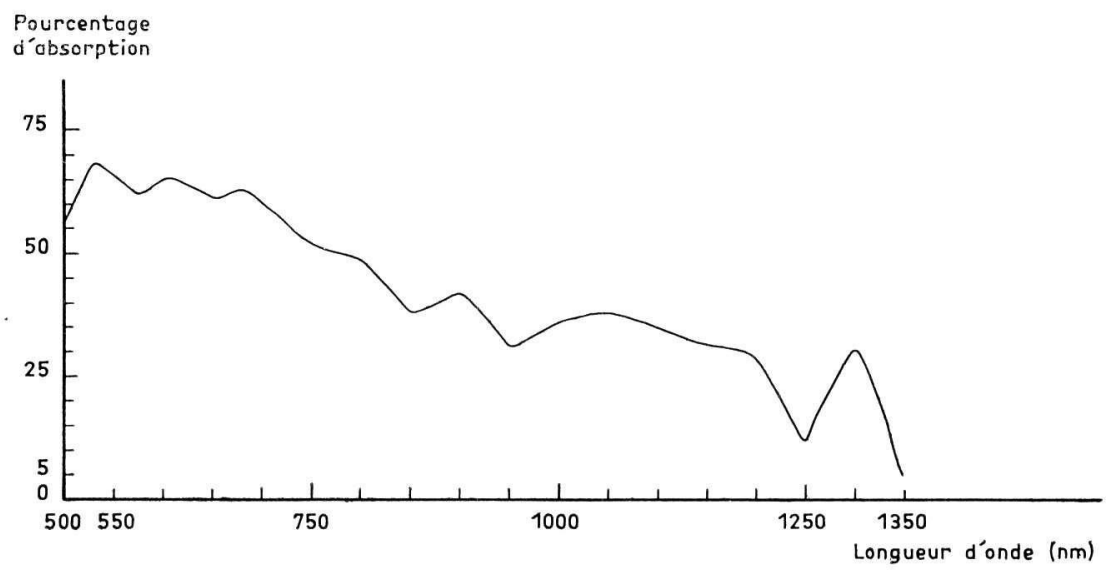

a

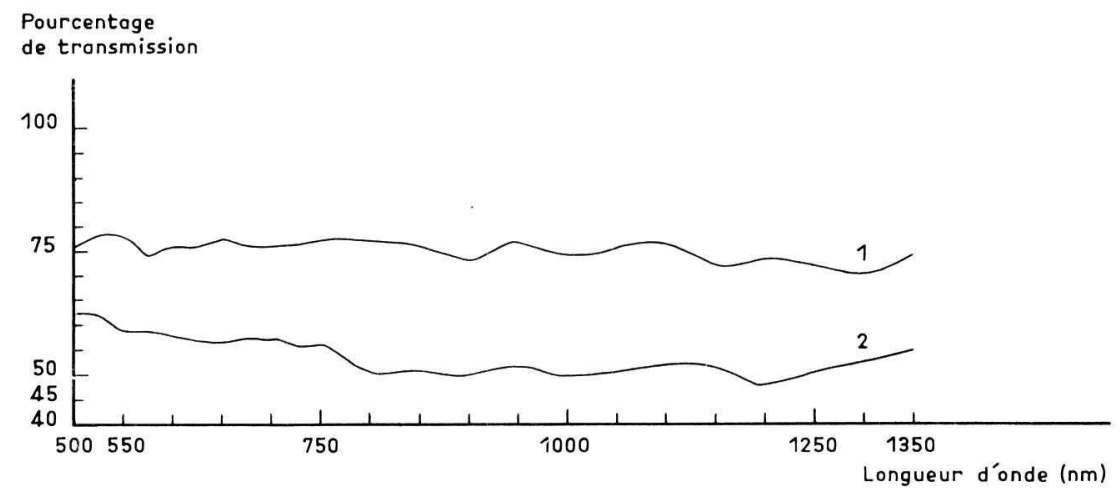

b

FIG. 4. - Propriétés optiques des poussières de charbon.

a) Spectre d'absorption (env. $5 \mathrm{~g} \cdot \mathrm{m}^{-2}$ ).

b) Spectre de transmission : 1 env. $0,5 \mathrm{~g} \cdot \mathrm{m}^{-2}$.

2 env. 1 g.m $\mathrm{m}^{-2}$.

Spectral properties of coal dust.

a) Absorption spectrum (ca. $5 \mathrm{~g} \cdot \mathrm{m}^{-2}$ ).

b) Transmission spectrum : $1 \mathrm{ca} .0 .5 \mathrm{~g} \cdot \mathrm{m}^{-2}$. 2 ca. 1 g.m ${ }^{-2}$.

\section{Discussion}

Les empoussièrements artificiels que nous avons obtenus sont très proches des empoussièrements naturels que l'on rencontre autour de certaines usines (Keller, 1971). Contrairement à nos expériences précédentes (Auclair, 1976b), nous avons 
effectué ici un empoussièrement homogène, avec des particules de diamètre apparent compris entre 1 et 20 microns, et sans gros agglomérats. D'autre part, ayant effectué nos expériences pendant un temps très court, nous avons pu ainsi ne considérer que l'effet physique des poussières sur la photosynthèse, les poussières n'ayant pu avoir d'effet chimique notable.

On remarque (łabl. 1 et 2) que la photosynthèse nette est significativement inhibée par les poussières aux éclairements faibles, mais non aux éclairements plus élevés. Ce résultat peut se voir plus clairement sur la figure 5 : on a évalué le pourcentage d'inhibition due à la poussière, en calculant le rapport $P^{\prime} / P$, à chaque éclairement.

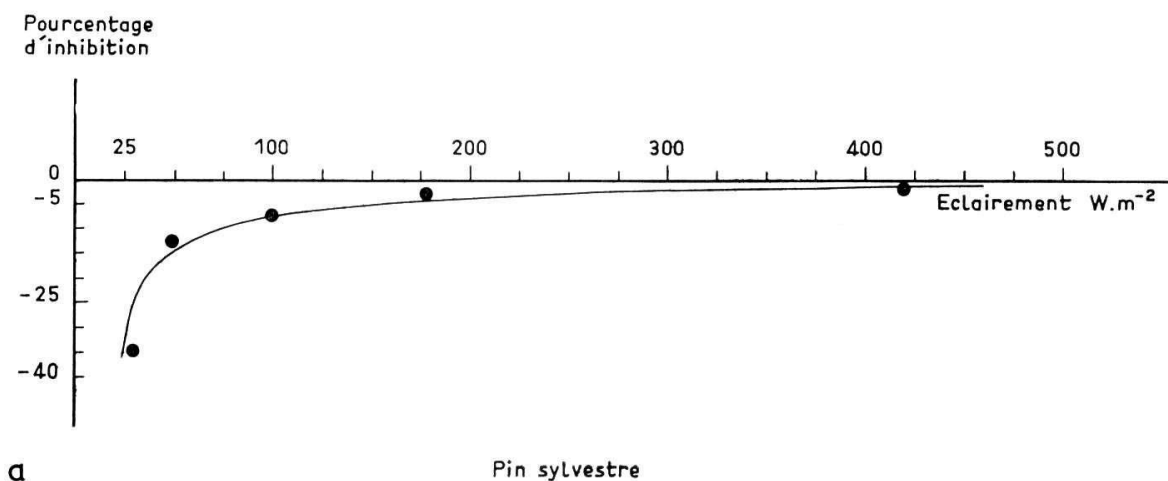

a

Pin sylvestre

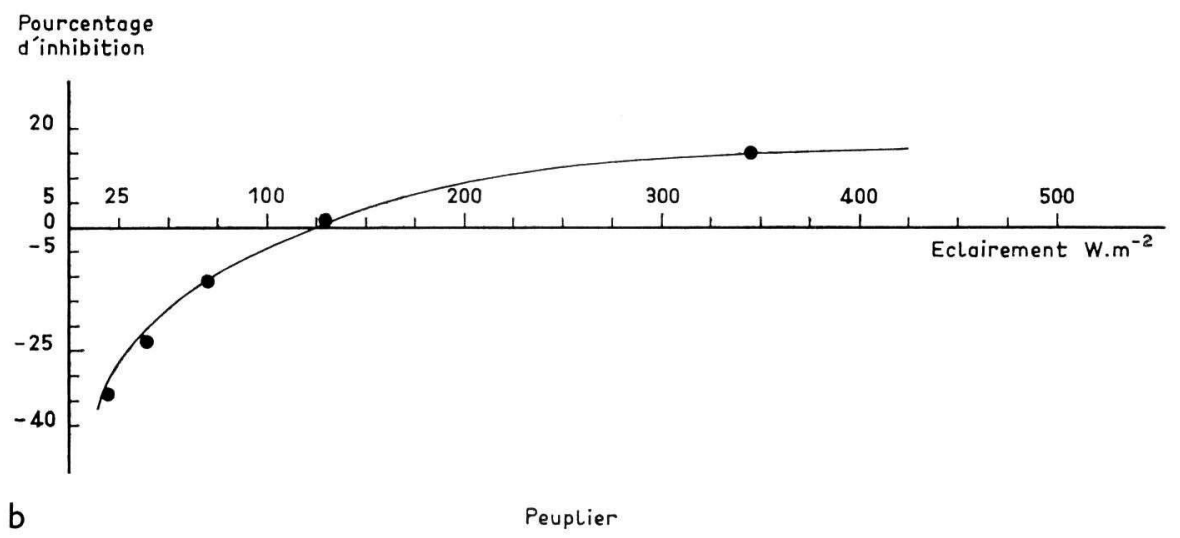

FIG. 5. - Pourcentage d'inhibition de la photosynthèse par la poussière.

a) Pin Sylvestre (env. $10 \mathrm{mg} \cdot \mathrm{g}^{-1}$ ).

b) Peuplier (env. $2 \mathrm{~g} \cdot \mathrm{m}^{-2}$ ).

Percentage of inhibition of photosynthesis, due to coal dusi.

a) Scots Pine (ca. $10 \mathrm{mg} \cdot \mathrm{g}^{-1}$ ).

b) Poplar $\left(c a .2 \mathrm{~g} \cdot \mathrm{m}^{-2}\right)$.

La photosynthèse est très fortement inhibée aux éclairements faibles et cette inhibition diminue en augmentant l'éclairement. Ceci confirme nos résultats préliminaires (Auclair, 1976b), sur des Épicéas. Les poussières semblent surtout capter de la lumière, diminuant l'énergie lumineuse utilisable pour la photosynthèse. Aux 
éclairements plus élevés, ce résultat ne peut être mis en évidence, la lumière n'étant plus le facteur limitant de la photosynthèse, d'autres éléments entrant en ligne de compte : transfert de $\mathrm{CO}_{2}$, réactions enzymatiques. Eveling (1967) a constaté un phénomène identique, des poussières inertes (kaolin, talc, silice) réduisant l'énergie lumineuse incidente d'environ 20 p. 100.

Il est à noter tableau 2 et figure 5 un résultat qui peut paraître surprenant, à l'éclairement maximal : la photosynthèse est plus forte chez les peupliers empoussiérés que chez les peupliers propres. Ceci peut s'expliquer de la manière suivante ; les poussières de charbon, absorbant du rayonnement infrarouge (fig. 4), transforment cette énergie en chaleur et réchauffent donc la feuille. Ce réchauffemerit entraîne une augmentation de la vitesse des réactions enzymatiques dans la feuille, ainsi que de la vitesse de diffusion du gaz carbonique. La photosynthèse est donc favorisée. D'autres réactions métaboliques sont également favorisées, ainsi que la transpiration. Mais, dans nos conditions expérimentales favorables (très bonne alimentation en eau, humidité relative moyenne), l'équilibre hydrique n'est pas modifié considérablement.

Ce phénomène peut se produire dans la nature, mais, en général tous les facteurs ne sont pas aussi favorables et un léger déficit hydrique peut entraîner un dessèchement accentué par l'augmentation de température.

La différence observée entre les figures $5 a$ et $5 b$ peut être due à une différence de morphologie foliaire : les feuilles de conifères sont plus épaisses, compactes et rigides que celles des feuillus. D'autre part, elles possèdent une cire cuticulaire très épaisse (Thair et Lister, 1975). Tous ces facteurs réunis peuvent entraîner un fort gradient de température, puisque l'éclairement est essentiellement incident. L'élévation de température due aux poussières n'agirait donc que sur un faible volume de l'aiguille, empêchant ainsi le phénomène observé sur le Peuplier.

Cette différence peut être simplement due à la différence d'empoussièrement : $10 \mathrm{mg} \cdot \mathrm{g}^{-1}$ pour les aiguilles et $80 \mathrm{mg} \cdot \mathrm{g}^{-1}$ pour les feuilles. Notons cependant que ces valeurs sont difficilement comparables, s'appliquant à des feuilles de morphologie très différente.

D'autres hypothèses sur l'augmentation de la photosynthèse aux éclairements forts peuvent être émises : modifications de l'ouverture stomatique ou de la perméabilité épidermique par un effet mécanique. Ricks et Williams (1974) ont constaté sur des feuillus une inhibition de la fermeture stomatique par des particules solides. Leurs observations microscopiques montrent des poussières obstruant partiellement l'ostiole, empêchant sa fermeture normale (à l'obscurité). Sur des conifères (Pin sylvestre) Gauthier et al. (1974) ont effectué des études semblables et ont montré que les poussières étaient fixées sur des bâtonnets de cire entourant la cavité sus-stomatique et ne pouvaient ainsi atteindre l'ostiole.

Mais, les stomates étant normalement ouverts aux éclairements considérés, il ne semble pas qu'une inhibition de leur fermeture puisse expliquer une augmentation de la photosynthèse.

Eveling (1967 ef 1972) a observé une augmentation de la transpiration sur des plantes ayant subi un très fort empoussièrement par des poussières inertes. II a constaté que ce phénomène était dû à une augmentation de la perméabilité épidermique, causée par le contact des poussières avec l'épiderme et non par leur compo- 
sition chimique. Un tel phénomène peut également expliquer des différences d'activité photosynthétique.

La figure 4 confirme les résultats constatés pour la photosynthèse : les poussières absorbent de l'énergie dans le spectre visible, ainsi que dans l'infrarouge. Dans le cas des poussières de charbon, la lumière transmise est diminuée de manière uniforme sur tout le spectre étudié. Une modification métabolique pourrait avoir lieu dans le cas de poussières transmettant à une longueur d'onde donnée (poussières de couleur), mais ceci n'a pas lieu avec les poussières étudiées, de couleur noire.

L'imprécision sur la mesure des propriétés spectrales des feuilles ne permet pas de formuler de conclusion sur ce point (fig. 3).

Nous avons obtenu une très grande dispersion des résultats, tant sur les Pins sylvestres (provenance génétique), que sur les Peupliers (clone). Ce résultat est en concordance avec les observations des forestiers (Lemoine, communication personnelle) qui observent une certaine variabilité (débourrement, croissance, etc.) au sein d'un même clone. Ceci semble lié à la position ou aux dimensions de la bouture lors de son prélèvement.

\section{Conclusion}

Nous avons étudié ici les effets physiques directs des poussières sur la photosynthèse, à des empoussièrements moyens, pour des arbres de morphologie différente : Pins sylvestres à aiguilles rigides, dont les stomates sont encastrés dans une chambre sus-stomatique, et Peupliers, feuillus à feuilles larges. Aux éclairements faibles, lorsque l'énergie lumineuse est le facteur limitant, la photosynthèse est assez fortement diminuée. Ceci est lié au phénomène d'interception de la lumière par les particules.

Aux éclairements forts, par contre, il n'y a pas d'inhibition. Le facteur limitant est le gaz carbonique. Les poussières n'accentuent pas cet effet, ce qui montre qu'elles n'empêchent pas l'arrivée de gaz carbonique en bouchant les stomates.

Nous n'avons pas pu effectuer de mesures sur la respiration à l'obscurité. Son augmentation (due à l'accroissement de la perméabilité épidermique, Eveling, 1972) annulerait les effets favorables des poussières aux éclairements forts : la matière accumulée pendant la journée serait ainsi perdue par respiration la nuit.

Nos résultats montrent que, s'ajoutant à un effet chimique possible à long terme, les poussières ont un effet notable sur la photosynthèse en diminuant l'énergie lumineuse utilisable par la plante. Ceci peut se traduire par une perte de production comme l'ont constaté Devaux et Le Bourhis (1974) sur un peuplement de Pins d'Alep'.

A des éclairements dépassant $100 \mathrm{~W} \cdot \mathrm{m}^{-2}$ (ce qui correspond à une journée par temps clair), il n'y a aucun effet notable, mais à des éclairements inférieurs (temps couvert, matin, soir, sous couvert forestier), on peut obtenir une diminution importante de la photosynthèse. Ces résultats nous donnent une idée de l'effet physique que peuvent avoir les poussières sur la végétation à travers la photosynthèse. Mais, dans la majorité des cas, les poussières affectent les plantes par leur composition chimique, pouvant ainsi entraîner des nécroses, dessèchements et même parfois la mort (Auclair, 1976a et 1976b). 


\author{
Summary \\ Effects of dust on photosynthesis \\ II. Effects of particulate matter on photosynthesis \\ of Scots Pine and Poplar
}

To study the physical effects of particulate matter on vegetation, photosynthesis was measured in a controlled gas-exchange chamber on Scots Pine and Poplar plantlets, before and after spraying with coal dust. The results were illustrated, using the schematic model :

$$
m P^{2}-\left(\alpha E+P_{\infty}\right) P+\alpha E P_{\infty}=0 .
$$

At high levels of illumination there was no inhibition of photosynthesis, on the contrary, Poplar seems to have a higher assimilation capacity after dusting. A possible effect on stomata or on leaf temperature is discussed. For low and medium light energies coal dust inhibits $\mathrm{CO}_{2}$ assimilation. This result is confirmed by the study of the spectral properties of the dust : it absorbs part of the incident light, and transmits only a fraction of the energy. Thus the maximum efficiency of incident light energy conversion is reduced, at low incident light energies.

\title{
Références bibliographiques
}

AUCLAIR D., 1974. Etude physiologique de deux espèces de Conifères : Abies alba (Miller) et Picea abies (L.) en relation avec leur capacité photosynthétique primaire. Rapport de D.E.A., Université d'Orsay, Paris XI, 9 p.

AUCLAIR D., 1976a. «Effets des Particules sur la Végétation », in Pollution par les particules émises dans l'atmosphère. Monographies scientifiques, série "Environnement », la Documentation française, Paris.

AUCLAIR D., 1976b. « Effets des Poussières sur la Photosynthèse. I. Effets des poussières de Ciment et de Charbon sur la photosynthèse de l'Epicéa. » Ann. Sci. For. 33 (4), 247-255.

CHARTIER P., 1966. «Etude théorique de l'assimilation brute de la Feuille. » Ann. Physiol. Vég., 8 (3), 167-195.

DARLEY E. F., 1966. "Studies on the Effect of Cement-kiln dust on Vegetation. ". J. Air Pollut. Control Assoc. 16 (3), 145-150.

DEVAUX J. P., LE BOURHIS M., 1974. «Effets phytotoxiques des poussières émises par une carrière de calcaire. Etude de la perte de production ligneuse d'une population de Pinus halepensis (Mill.). » Rev. Biol. Ecol. Méd., 1 (2), 41-52.

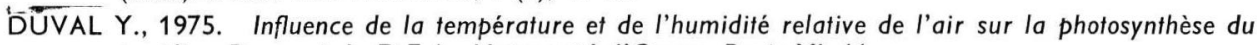
peuplier. Rapport de D.E.A., Université d'Orsay, Paris XI, 11 p.

EVELING D. W., 1967. Physical effects of particulate sprays on leaf physiology. Thèse Ph. D., London.

EVELING D. W., 1972. "Similar effects of suspensions of copper oxychloride and kaolin on sprayed A. leaves. » Ann. Appl. Biol., 70, 245-249.

GAUTHIER D., GARREC J. P., BELOT Y., FOURCY A., 1974. Effets mécaniques des Dépôts de Poussières sur les échanges gazeux de Pinus sylvestris L. Publication interne, C.E.A., Département de Protection, Laboratoire des Transferts atmosphériques, $9 \mathrm{p}$.

KELLER T., 1971. «Wälder, Parkbäume-und Luftverunreinigungen. » Die Schweizer Gemeinde, 8 (32).

RICKS G. R., WILLIAMS R. J. H., 1974. «Effects of Atmospheric Pollution on Deciduous Woodland, Part 2 : Effects of Particulate Matter upon Stomatal Diffusion Resistance in Leaves of Quercus petraea (Mattuschka) Leibl. » Environ. Pollut., 6, 87-109.

ROUX C., TOMASSONE R., 1973-1974. Moindres carrés non linéaire HAUS 59. Publication interne I.N.R.A., laboratoire de Biométrie du C.N.R.Z., Documents 73/20 et 74/04, 47 p.

THAIR B. W., LISTER G. R., 1975. The distribution and fine structure of the epicuticular leaf wax of Pseudotsuga menziezii. Can. J. Bot., 53 (10), 1053-1071. 